\title{
Intruder States in Multireference Perturbation Theory: The Ground State of Manganese Dimer
}

\author{
CRISTOPHER CAMACHO, ${ }^{1}$ HENRYK A. WITEK, ${ }^{1}{ }^{\text {SHIGEYOSHI YAMAMOTO }}{ }^{\mathbf{2}}$ \\ ${ }^{1}$ Institute of Molecular Science and Department of Applied Chemistry, National Chiao Tung \\ University, 1001 Ta-Hsueh Road, Hsinchu 30010, Taiwan \\ ${ }^{2}$ Faculty of Liberal Arts, Chukyo University, 101-2 Yagoto-Honmachi, Showa-ku, Nagoya, Aichi \\ 466-8666, Japan
}

Received 10 May 2008; Accepted 13 June 2008

DOI 10.1002/jcc.21074

Published online 5 August 2008 in Wiley InterScience (www.interscience.wiley.com).

\begin{abstract}
A detailed analysis of a severe intruder state problem in the multistate multireference perturbation theory (MS-MRPT) calculations on the ground state of manganese dimer is presented. An enormous number of detected intruder states $(>5000)$ do not permit finding even an approximate shape of the $X^{1} \Sigma_{g}^{+}$potential energy curve. The intruder states are explicitly demonstrated to originate from quasidegeneracies in the zeroth-order Hamiltonian spectrum. The electronic configurations responsible for appearance of the quasidegeneracies are identified as single and double excitations from the active orbitals to the external orbitals. It is shown that the quasidegeneracy problem can be completely eliminated using shift techniques despite of its severity. The resultant curves are smooth and continuous. Unfortunately, strong dependence of the spectroscopic parameters of the $X^{1} \Sigma_{g}^{+}$state on the shift parameter is observed. This finding rises serious controversies regarding validity of employing shift techniques for solving the intruder state problem in MS-MRPT. Various alternative approaches of removing intruder states (e.g., modification of the basis set or changing the active space) are tested. None of these conventional techniques is able to fully avoid the quasidegeneracies. We believe that the MS-MRPT calculations on the three lowest $A_{g}$ states of manganese dimer constitute a perfect benchmark case for studying the behavior of MRPT in extreme situations.
\end{abstract}

(C) 2008 Wiley Periodicals, Inc. J Comput Chem 30: 468-478, 2009

Key words: perturbation theory; intruder states; MCQDPT; CASPT2

\section{Introduction}

Theoretical determination of potential energy curves for metal dimers is very challenging because of a large number of lowlying electronic states and highly multiconfigurational character of the corresponding wave functions. This statement is particularly true for transition metals, lanthanides, and actinides because of the existence of many atomic terms arising from not fully occupied atomic $d$ and $f$ orbitals. A molecule that possess remarkable computational difficulties is the manganese dimer. Until recently, ${ }^{1}$ not only the shape of the potential energy curve was not known, but also the multiplicity or angular momentum of the ground state was a subject of controversy. ${ }^{1-17}$ This situation was mainly caused by a truly multideterminantal character of the ground state wave function and the dispersion being the primary force binding the two manganese atoms. Many DFT calculations were reported on $\mathrm{Mn}_{2},{ }^{6-16}$ but they led to diametrically different conclusions about the equilibrium bond length and the electronic character of the ground state, depending on the functional and the approach used. The complete active space self-consistent field (CASSCF) method predicts that the ground state PES is unbound. ${ }^{4}$ Accounting for dynamical correlation using the multireference configuration interaction (MRCI) method is prohibitively expensive; preliminary MRCI calculations yield repulsive curves.* The potential energy curves determined ${ }^{1,2}$ using second-order multireference perturbation theory (MRPT) predict that manganese dimer is a weakly bound van der Waals molecule and that the ground state of $\mathrm{Mn}_{2}$ is ${ }^{1} \Sigma_{g}^{+}$. These theoretical predictions agree well with experimental findings.

Additional Supporting Information may be found in the online version of this article.

Correspondence to: H. Witek; e-mail: hwitek@mail.nctu.edu.tw

Contract/grant sponsor: National Science Council; contract/grant number: NSC 96-2113-M-009-022-MY3

Contract/grant sponsor: Ministry of Education of Taiwan (MOE-ATU Project)

*Our preliminary MRCI/CASSCF calculations used the (10e,10o) active space. For an incomplete MRCI/MCSCF model space, 1500 leading CSFs have been selected from the $(12 \mathrm{o}, 14 \mathrm{e})$ active space. Both sets of calculations yield repulsive curves. Larger MRCI calculations have not been feasible in our computing environment (8CPUs machine with 32 GB RAM). 
At the moment, MRPT is the only way of obtaining physically meaningful estimates of the ground state potential energy surface.

In the context of the discussion earlier, it is particularly interesting that the reported ${ }^{1}$ PES calculations for the low-lying states of manganese dimer using second-order multistate multireference perturbation theory (MS-MRPT) suffer from the intruder state problem, and it was necessary to employ the intruder state avoidance (ISA) technique ${ }^{18}$ to ensure smooth character of the calculated curves. To make the reader more familiar with the intruder state problem, let us recall the second-order energy correction in Rayleigh-Schrödinger perturbation theory given by

$$
E_{\alpha}^{(2)}=\sum_{k} \frac{\langle\alpha|\hat{H}| k\rangle^{2}}{E_{\alpha}^{(0)}-E_{k}^{(0)}},
$$

where $|\alpha\rangle$ is a reference wave function, $|k\rangle$ denotes all singly and doubly excited intermediate states, and $E_{\alpha}^{(0)}$ and $E_{k}^{(0)}$ are the zeroth-order energies of the reference and intermediate states, respectively. Intruder states are defined as those of the intermediate states that have their zeroth-order energies quasidegenerate with the zeroth-order energy of the reference state, $E_{\alpha}^{(0)} \approx E_{k}^{(0)}$. Presence of such states results in small energy denominators in eq. (1) and, consequently, leads to unphysically large-either positive or negative-value of $E_{\alpha}^{(2)}$. In PES calculations, intruder states usually manifest themselves as discontinuities. Because the character of the discontinuities is usually very local, they are frequently overlooked owing to large separations between the points defining PES. When the number of such singularities is considerable, or when a large number of points is requested, the possibility of their accidental encountering can be large. Consequently the calculated PES is not smooth and displays peculiar local features. We believe that this is a serious problem of MRPT because in practice it leads to reduction of its popularity. Many researchers-especially those not very familiar with mathematical details of perturbation theory-after applying MRPT to their problems and obtaining nonsmooth PES, feel discouraged and decide to switch to a different computational method. Various techniques have been developed ${ }^{18-21}$ to overcome the intruder state problem. Most of them employ a real or imaginary shift operator that modifies the zeroth-order energies of the reference or intermediate states and leads to a modified second-order energy correction given by

$$
E_{\alpha}^{(2)}=\sum_{k} \frac{\langle\alpha|\hat{H}| k\rangle^{2}}{E_{\alpha}^{(0)}-E_{k}^{(0)}+\Delta},
$$

where $\Delta$ is a shift parameter that may depend on $k$. Intruder state removal shift techniques are available in most of quantum chemistry packages $^{22-24}$ and are conventionally used to ensure smooth character of the calculated potential energy surfaces. ${ }^{25-39}$ It is important to stress here that the shift techniques are not a panacea for all problems originating from a selection of poor partitioning in MRPT. It may happen that using these techniques may lead to neglect of substantial portion of dynamical correlation or skipping some potentially important intermediate states in the perturbation expansion that otherwise would interact strongly with the reference wave function.
Intruder states are observed in second-order MRPT mainly in calculations for high-lying electronic states. ${ }^{18,19,40}$ It is rather unusual to observe zeroth-order spectrum quasidegeneracies already for the ground state. ${ }^{19,21}$ Therefore, we have decided to perform a detailed study of the previously reported ${ }^{1}$ multistate second-order multiconfigurational quasidegenerate perturbation theory (MCQDPT) potential energy curve for the ground state of the manganese dimer to elucidate the character and the origin of the intruder states and to test various standard techniques of their removal (change of active space, change of basis set, different number of coupled states in the MCQDPT formalism, and various schemes of orbital canonicalization). Another important aspect studied here is the dependence of the calculated potential energy curves and the spectroscopic parameters of $\mathrm{Mn}_{2}$ on the value of the shift parameter used to remove intruder states. To show that the reported problems are not only specific to the MCQDPT method, additional calculations are performed with different variants of MRPT: fully- and partially-contracted second-order complete active space perturbation theory (CASPT2). The results of these investigations - that by far have exceeded our expectations-are presented here.

\section{Computational Details}

The ${ }^{1} \Sigma_{g}^{+}$ground state potential energy curve of $\mathrm{Mn}_{2}$ has been studied in details using MCQDPT ${ }^{41}$ implemented in the GAMESS $^{22}$ quantum chemistry package. More than 4000 singlepoint MCQDPT energy calculations have been performed to provide a consistent description of the studied PES. All-electron $(18 s 15 p 8 d 4 f 2 g) /[7 s 6 p 4 d 4 f 2 g]$ basis set, $(14 \mathrm{e}, 12 \mathrm{o})$ complete active space (14 active electrons in 12 active orbitals originating from the $3 d$ and $4 s$ AOs), and other computational details have been adopted from the previous study by Yamamoto et al. ${ }^{1}$ The three lowest roots of symmetry $A_{g}$ (corresponding to the two lowest ${ }^{1} \Sigma_{g}^{+}$states and a single component of the ${ }^{1} \Delta_{g}$ state) are used to construct the effective MCQDPT Hamiltonian. Two orbital canonicalization schemes are tested: state-averaged (for the three studied states) and state-specific (only for the ground state). The $1 s, 2 s$, and $2 p$ orbitals of each manganese atom are not correlated. Analogous calculations are performed with two alternative versions of MRPT, the CASPT2 programs implemented in the MOLCAS ${ }^{24}$ and MOLPRO $^{23}$ quantum chemistry programs. In the MOLPRO version of CASPT2 serious computational problems occur if the number of doubly-occupied and active electrons approaches 30 (as it is in our case). Therefore, the CASPT2/MOLPRO calculations have not included any of the doubly occupied orbitals in the perturbation treatment. The following shift techniques have been tested for their ability of removing intruder states: the intruder state avoidance (ISA) technique, ${ }^{18}$ the real shift technique of Roos and Andersson, ${ }^{21}$ and the imaginary shift technique of Forsberg and Malmqvist. ${ }^{19}$ Simultaneously, various traditional approaches of avoiding the intruder state problem are tested; more detailed information is given in the next Section. Exemplary GAMESS, MOLPRO, and MOLCAS input files for the CASSCF and MRPT calculations are given in Auxiliary Materials to facilitate reproducing our results. Numerical data used to prepare the presented plots are available in electronic form in Auxiliary Materials as GRACE input files. 


\section{Results and Discussion}

\section{MCQDPT Potential Energy Curves}

Previously reported ${ }^{1}$ MCQDPT potential energy curve for the lowest ${ }^{1} \Sigma_{g}^{+}$state of $\mathrm{Mn}_{2}$ was apparently smooth and continuous. However, the regular and uniform character of this curve could only be recovered after employing the ISA technique ${ }^{18}$ in the MCQDPT calculations. The original MCQDPT PES calculated without the ISA technique shows numerous discontinuities related to quasidegeneracies in the zeroth-order MCQDPT Hamiltonian. The extent and magnitude of distortions introduced in the MCQDPT PES by the intruder states have motivated us to present here a detailed, spatially-resolved study of this curve.

Diagonal elements of the effective MCQDPT Hamiltonian corresponding to the two lowest ${ }^{1} \Sigma_{g}^{+}$states of manganese dimer are shown in Figure 1 as a function of internuclear separation. Analogous data for the distance-dependent coupling between these states, which corresponds to the only nonvanishing off-diagonal elements of the $3 \times 3$ MCQDPT effective Hamiltonian, is shown in Figure 2. The upper panels of both plots give an overview of the computed curves between 1.9 and $4.0 \AA$. Each curve contains an enormous number of discontinuities - estimated to be larger than 5000-at distances shorter than $3.2 \AA$. The abundance of singularities and the magnitude of changes introduced in their vicinity do not permit finding even an approximate shape of the computed curves. This is best visible in the middle and bottom panels of Figures 1 and 2 that show two subsequent magnifications of a small segment of the upper panels. The curves in Figures 1 and 2 may seem erratic at the first sight, but the performed magnifications show that in fact the curves are locally smooth and show a typical up-down branching ${ }^{18}$ around every singular geometry. It must be stressed that even at nonsingular geometries (e.g., $2.3104 \AA$ ) the error introduced by intruder states can be quite substantial, which effectively prevents guessing even an approximate shape of the curves.

The MCQDPT potential energy curves are obtained by diagonalizing the $3 \times 3$ MCQDPT effective Hamiltonian for every considered internuclear separation. It is clear that this procedure cannot be a remedy for the intruder states present in the diagonal and off-diagonal elements of the effective Hamiltonian. As expected, the calculated MCQDPT curves display an abundance of singularities that completely prevent one from finding the actual shape of the potential energy curves. A fragment of the calculated MCQDPT potential energy curves between 2.3071 and $2.3284 \AA$ is shown in Figure 3. It is amazing that only the presented small segment (approximately $0.02 \AA$ ) of the total PESs contains more than 35 singularities! This example shows that the multistate $(12 \mathrm{o}, 14 \mathrm{e})$ MCQDPT calculations without the shift corrections are completely meaningless for the lowest ${ }^{1} \Sigma_{g}^{+}$state of $\mathrm{Mn}_{2}$. In a practical computational scheme, usually only a small number of probe geometries (say 30) are used to determine the shape of the whole PES. In the case studied by us such an approach inevitably would lead to an almost random shape of the calculated curve. After employing the ISA shift technique with the shift parameter equal to 0.02 , it is possible to completely remove all the discontinuities from all the three studied curves (see the upper panel of Fig. 4). Unfortunately, a closer inspection of the ISA-MCQDPT curves (or more general: shifted MRPT curves) shows a very undesirable feature. The shape of the ground state PES and the corresponding spectroscopic parameters depend strongly on the employed value of the shift parameter. These findings rise serious controversy regarding mathematical validity of applying the shift techniques for solving the intruder state problem in MRPT. We discuss this phenomenon further in Section "Failure of Shift Techniques".

\section{CASPT2 Potential Energy Curves}

To verify that the reported intruder state problem is not a specific feature of the MCQDPT method, we have performed analogous calculations using two alternative versions of multistate MRPT: the partially-contracted CASPT2 method implemented in the MOL$\mathrm{PRO}^{23}$ package and the fully-contracted CASPT2 method implemented in the MOLCAS ${ }^{24}$ package. The calculations have been performed using a standard canonical definition of the Fock operator without additional shift techniques; analogous calculations employing shift techniques are discussed in Section "Failure of Shift Techniques". No solution of the CASPT2 equations could be obtained with the CASPT2/MOLPRO program at any of the selected 27 test geometries between 1.9 and $5.4 \AA$. The norm of the CASPT2 wave function grows unlimitedly clearly indicating presence of intruder states. The CASPT2 potential energy curves obtained with the CASPT2/MOLCAS program are shown in the lower panel of Figure 4. Numerous intruder states are detectedespecially for the higher two states-but it is clear that the intruder state problem in CASPT2 is far less serious than in MCQDPT (a direct comparison of both sets of curves is somewhat impeded by the fact that the MCQDPT curves are plotted using more than 4000 single point energies, while for CASPT2, only about 450 energies are computed). Less severe problems with intruder states in CASPT2 are easily understood if one takes into account much smaller number of intermediate states used to construct the CASPT2 first-order wave function. The minimum of the CASPT2/MOLCAS ground state PES is located approximately at $3.2 \AA$; a number of intruders present around this geometry prohibit determining other spectroscopic parameters. Similarly to MCQDPT, employing shift techniques allows for obtaining smooth potential energy curves with both versions of CASPT2. (See Figs. 4 and 6.) Unfortunately, again the shape of the shifted CASPT2 potential energy curves and the computed spectroscopic parameters depend strongly on the value of the shift parameter; the character of this dependence is discussed in details in Section "Failure of Shift Techniques".

\section{Analysis of Intruder States in the MCQDPT Calculations}

The first-order interacting space (FOCI) used to account for dynamical correlation in the MCQDPT method is spanned by all singly and doubly excited CSFs with respect to the CASSCF configuration state functions. These excited CSFs-often referred to as intermediate states - can be classified into eight different groups, depending on the number of excited electrons and their original and final locations. ${ }^{42,43}$ Dividing the molecular orbitals into the inactive (I), active (A), and external (E) subspaces allows for symbolic representation of these eight classes as: $\mathrm{I} \rightarrow \mathrm{A}, \mathrm{II} \rightarrow \mathrm{AA}$ (internal excitations), $\mathrm{I} \rightarrow \mathrm{E}$, $\mathrm{A} \rightarrow \mathrm{E}, \mathrm{II} \rightarrow \mathrm{AE}$ (semiinternal excitations), $\mathrm{II} \rightarrow \mathrm{EE}, \mathrm{IA} \rightarrow \mathrm{EE}$, and $\mathrm{AA} \rightarrow \mathrm{EE}$ (external excitation). Here, the notation $\mathrm{II} \rightarrow \mathrm{AE}$ should be understood as an excitation of two inactive electrons into single active and single external orbital. Number of excited electronic 


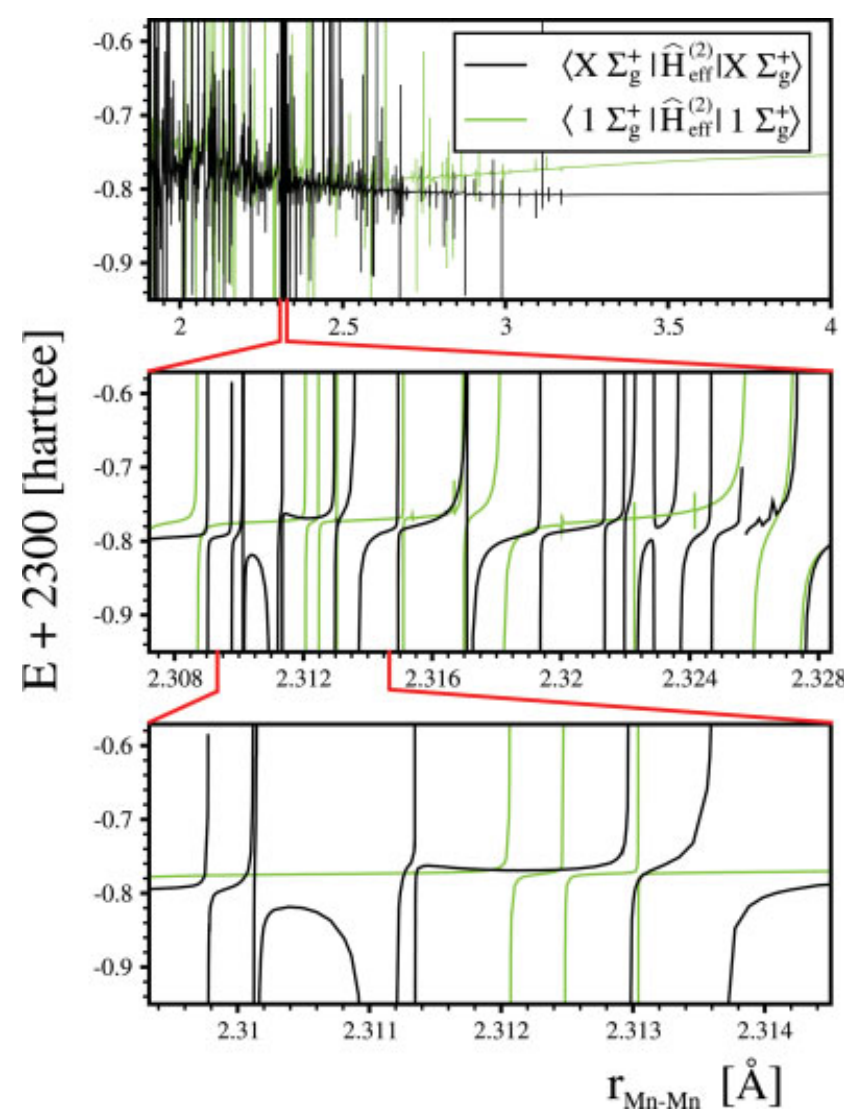

Figure 1. Distance-dependent diagonal elements of the MCQDPT effective Hamiltonian $\hat{H}_{\text {eff }}^{(2)}$ for the ground and the first excited ${ }^{1} \Sigma_{g}^{+}$ states of $\mathrm{Mn}_{2}$.

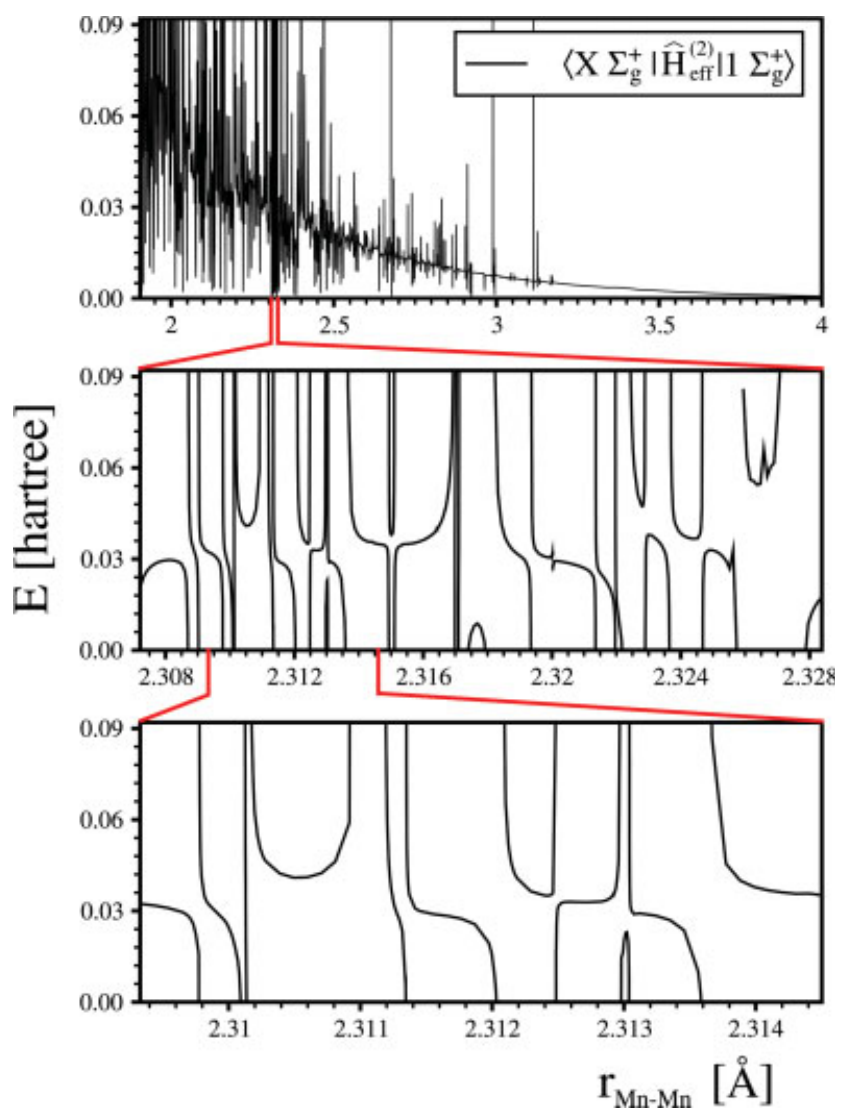

Figure 2. Distance-dependent off-diagonal elements of the MCQDPT effective Hamiltonian $\hat{H}_{\text {eff }}^{(2)}$ between the ground and the first excited ${ }^{1} \Sigma_{g}^{+}$ states of $\mathrm{Mn}_{2}$.

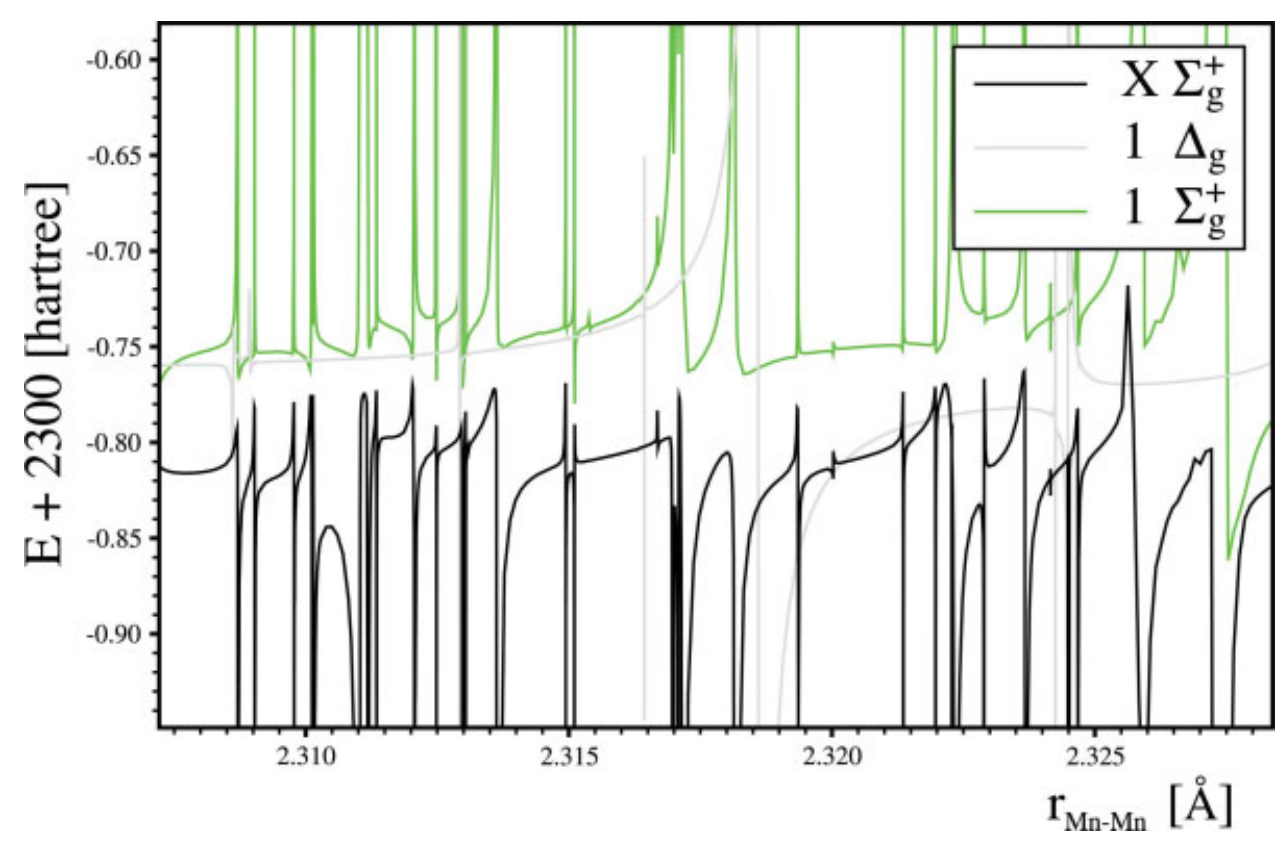

Figure 3. A fragment of the MCQDPT potential energy curves for the three lowest $A_{g}$ roots. 
configurations in each class is given in Table 1. It is natural to ask which of those excitation classes are responsible for the occurrence of intruder states in our MCQDPT calculations. To answer this question, we plot in Figure 5 the zeroth-order energies $E_{k}^{(0)}$ of all the intermediate states together with the distance-dependent zeroth-order energy $E_{\alpha}^{(0)}$ of the reference state as a function of internuclear separation. The zeroth-order energies are given separately for each of the eight classes of excitations. For six out of the eight classes, $E_{k}^{(0)}$ are well separated from $E_{\alpha}^{(0)}$. However, for two classes of excitations, $\mathrm{A} \rightarrow \mathrm{E}$ and $\mathrm{AA} \rightarrow \mathrm{EE}, E_{\alpha}^{(0)}$ is completely dipped in the FOCI section of the zeroth-order Hamiltonian spectrum. For $\mathrm{A} \rightarrow \mathrm{E}$, the number of detected quasidegeneracies at various geometries is equal to 3486, which accounts for approximately $0.3 \%$ of the total number of the $\mathrm{A} \rightarrow \mathrm{E}$ excited electron configurations. For $\mathrm{AA} \rightarrow \mathrm{EE}$, the number of detected quasidegeneracies at various geometries is equal to 1500 , which accounts for approximately $0.001 \%$ of the total number of the $\mathrm{AA} \rightarrow \mathrm{EE}$ excited electron configurations. Note that the actual number of intermediate CSFs responsible for appearance of intruder states is larger, because a single open-shell electronic

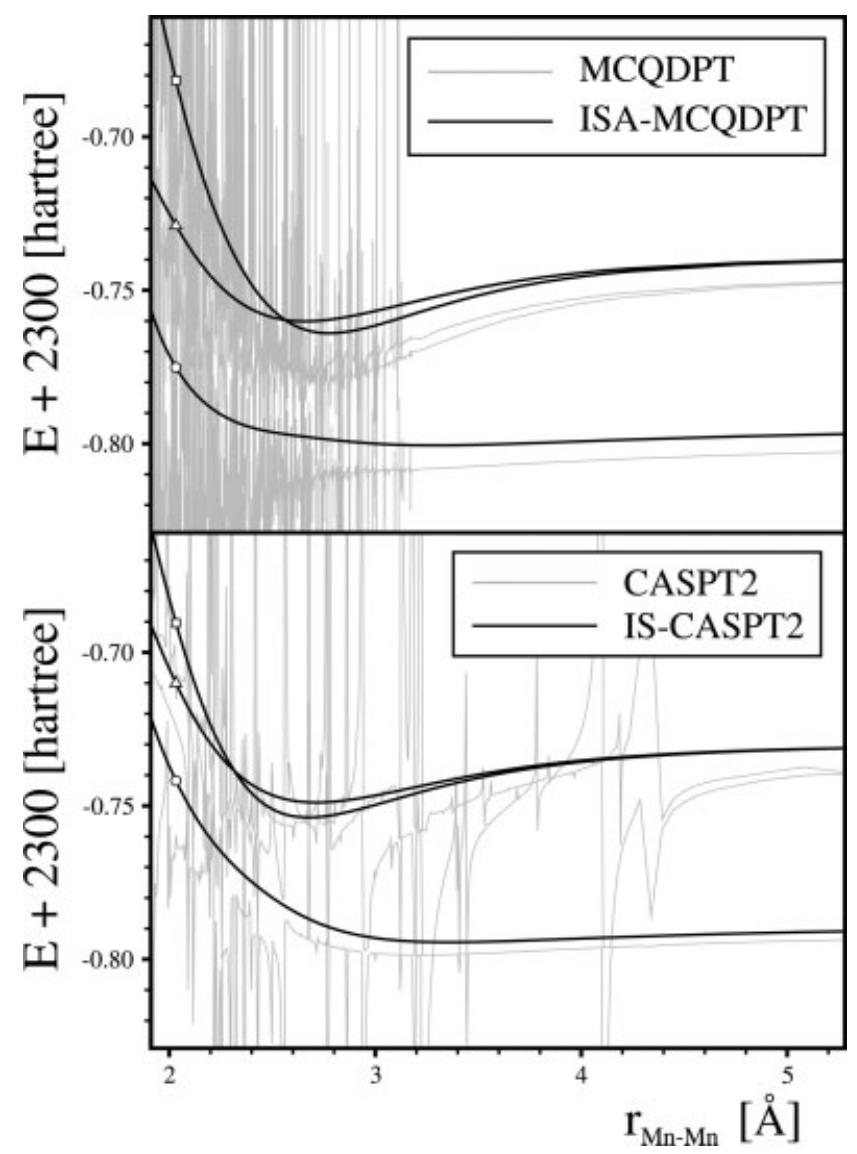

Figure 4. Comparison of the MCQDPT and ISA-MCQDPT (upper panel) and CASPT2 and IS-CASPT2 (lower panel) potential energy curves for the $X^{1} \Sigma_{g}^{+}(\bigcirc), 1^{1} \Sigma_{g}^{+}(\square)$, and $1^{1} \Delta_{g}(\triangle)$ states of $\mathrm{Mn}_{2}$. The value of the ISA shift parameter is 0.02 . The value of imaginary shift in the IS-CASPT2 calculations is 0.3 .
Table 1. Total Number of Electronic Configurations with the $A_{g}$ Symmetry for the Eight Possible Classes of FOCI Excitations. (For Details, See Text.) The Original CASSCF Space Corresponds to 10 Core, 8 Inactive, 12 Active, and 152 External Orbitals and 14 Active Electrons.

\begin{tabular}{cr}
\hline Subspace & Number of configurations \\
\hline $\mathrm{A} \rightarrow \mathrm{E}$ & $1,327,256$ \\
$\mathrm{AA} \rightarrow \mathrm{EE}$ & $107,367,376$ \\
$\mathrm{I} \rightarrow \mathrm{A}$ & 43,760 \\
$\mathrm{I} \rightarrow \mathrm{E}$ & $8,877,904$ \\
$\mathrm{IA} \rightarrow \mathrm{EE}$ & $809,420,160$ \\
$\mathrm{II} \rightarrow \mathrm{AA}$ & 129,028 \\
$\mathrm{II} \rightarrow \mathrm{AE}$ & $29,633,136$ \\
$\mathrm{II} \rightarrow \mathrm{EE}$ & $3,050,580,208$ \\
\hline
\end{tabular}

configuration may give rise to a larger number of CSFs via different spin coupling schemes. However, all such CSFs would manifest themselves as a single intruder state in PES because they share the same value of the zeroth-order energy.

A closer inspection of both excitation classes shows that the external orbitals responsible for the quasidegeneracies always correspond to the lowest virtual orbitals in each irrep. At short distances, the number of such low-lying external orbitals can be considerable; the detected intruder states are caused by excitations to MOs originating from the atomic $4 p, 5 p$, and $5 s$ orbitals. For longer distances, the observed quasidegeneracies are caused only by the atomic $4 p$ orbitals. This analysis shows that the standard method of avoiding intruder states by shifting the low-lying external orbitals to the active space is not applicable here owing to prohibitively large dimension of the resulting CAS problem.

It is important to mention that even if the zeroth-order energies of some of the intermediate states do not cross with that of the reference state, $E_{\alpha}^{(0)}-E_{k}^{(0)}$ can still be very small in some regions of PES. In such situations, the potential energy curve can exhibit strange local features (bubbles). ${ }^{\dagger}$ For $\mathrm{Mn}_{2}$, even if such peculiarities are present in the potential energy curve, they are effectively masked by the enormous number of singularities.

\section{Failure of Shift Techniques}

As can be seen from Figure 4, using shift techniques implies systematic shift of the calculated MRPT energies upward, which can be explained by partial cancellation of the dynamical correlation energy by larger value of denominators in the perturbation series. Even though this effect is rather small, ${ }^{\ddagger}$ it may have strong impact on calculated potential energy curves because the amount of the dynamical energy cancellation is different at various geometries. This behavior seems to have serious practical consequences. The calculated spectroscopic parameters (equilibrium

${ }^{\dagger}$ For an example of such a feature, see the curve for the $2^{3} \Delta$ state of $\mathrm{AgH}$ at 3.9 Å given in ref. 18 .

\#At $4.0 \AA$ A , i.e., at a distance not affected by intruder states, the ISA-MCQDPT method accounts for 99.4, 99.3, and 99.3\% of the MCQDPT dynamical correlation energy for $X^{1} \Sigma_{g}^{+}, 1^{1} \Sigma_{g}^{+}$, and $1^{1} \Delta_{g}$, respectively. 


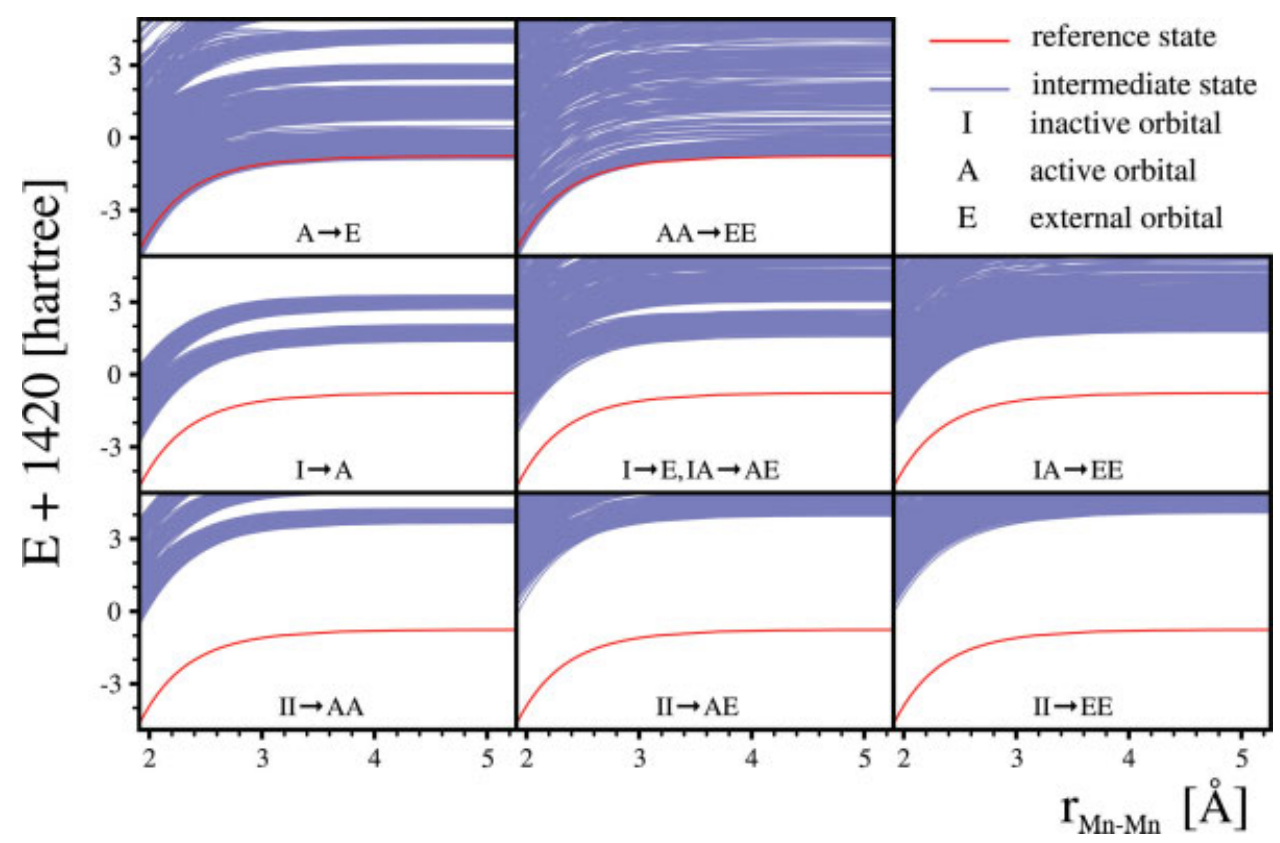

Figure 5. Zeroth-order MCQDPT energies of the reference state (dark red line) and the intermediate states (light blue line) for all the possible classes of single and double excitations spanning the FOCI space.

bond lengths, dissociation energies, harmonic vibrational frequencies, and excitation energies) depend strongly on the value of the shift parameter used to remove intruder states. In Table 2, we have collected various spectroscopic parameters for the ground state of $\mathrm{Mn}_{2}$ computed using the MCQDPT and CASPT2 method with various shift techniques: the intruder state avoidance (ISA) technique, ${ }^{18}$ the real shift (RS) technique of Roos and Andersson, ${ }^{21}$ and the imaginary shift (IS) technique of Forsberg and Malmqvist. ${ }^{19}$ Five different shift values are employed for each technique to visualize the scale of changes induced by varying the shift parameter. The corresponding ISA-MCQDPT, IS-CASPT2/MOLCAS, and RS-CASPT2/MOLPRO potential energy curves are shown in Figure 6 . The position of the minimum on each of the curves-marked with a symbol-is systematically shifted toward longer distances for larger value of the shift parameter. This behavior can be readily understood if one recollects that the CASSCF potential energy curve for the ground state of $\mathrm{Mn}_{2}$ is repulsive and that the larger value of shift effectively diminishes the amount of dynamical correlation recovered by MRPT; it is clear that for the infinite value of the shift parameter, the CASSCF curve should be reproduced. Analogous pattern can be observed for the other spectroscopic parameters presented in Table 2. Note that in the studied case it is impossible to predict unbiased values of spectroscopic parameters for the ground state of manganese dimer using MRPT with shift techniques.

\section{Alternative Methods of Removing Intruder States}

It is a common belief in the computational chemistry community that the intruder state problem in second-order MRPT can be solved in a number of ways. The most popular solution is including the low-lying external orbitals that are responsible for quasidegeneracies in the active space. Another solution is using shift techniques. Unfortunately, as we have shown earlier, these solutions are not applicable here. Later we discuss other alternative methods of avoiding intruder states in the MRPT calculations. Probably, the simplest way is to choose a different set of geometries defining PES in hope to avoid the singular geometries. Unfortunately, in the three-state MRPT calculations on $\mathrm{Mn}_{2}$ virtually all geometries are affected by some-more or less local-intruder states. Another way is to modify the one-electron basis set used in the calculations, preferably by removing some of the diffuse functions. Sometimes it is also possible to change the set of MOs used in the MCQDPT calculations by performing appropriate state-averaging in the CASSCF calculations. Another possibility can be to enlarge/reduce the number of states in the effective MCQDPT Hamiltonian, which can modify both, the definition of the Fock operator and the set of canonical MOs. Finally, we mention here probably the most popular solution, i.e., employing other active space to define the CASSCF reference wave function. Later we discuss our results obtained when applying these techniques to the multistate MRPT calculations for the ground state of $\mathrm{Mn}_{2}$.

\section{Modification of Atomic Basis Set}

Our main objective here is to investigate the possibility of modifying the basis set for the manganese atom in a way that enables avoiding the intruder state problem in the multistate MCQDPT calculations for the ground state of $\mathrm{Mn}_{2}$. We request that the basis set modifications should not change the energy of the CASSCF reference wave function (or do it in a minimal degree). To this end, we have gradually kept removing some portion of the atomic basis set and inspected the effect of this operation on the CASSCF and MCQDPT curves for the ${ }^{1} \Sigma_{g}^{+}$of $\mathrm{Mn}_{2}$. We have been able to remove the $g, f$, and the two most diffuse $p$ atomic orbitals without significant alteration of the CASSCF curve. It has not been possible to modify the $s$ and $d$ segments of the basis set in the same manner. The MCQDPT 
Table 2. Spectroscopic Parameters for the Ground State of $\mathrm{Mn}_{2}$ Computed Using MRPT with Different Shift Techniques (For Details, See Text). Note Strong Dependence of the Presented Results on the Value of the Shift Parameter.

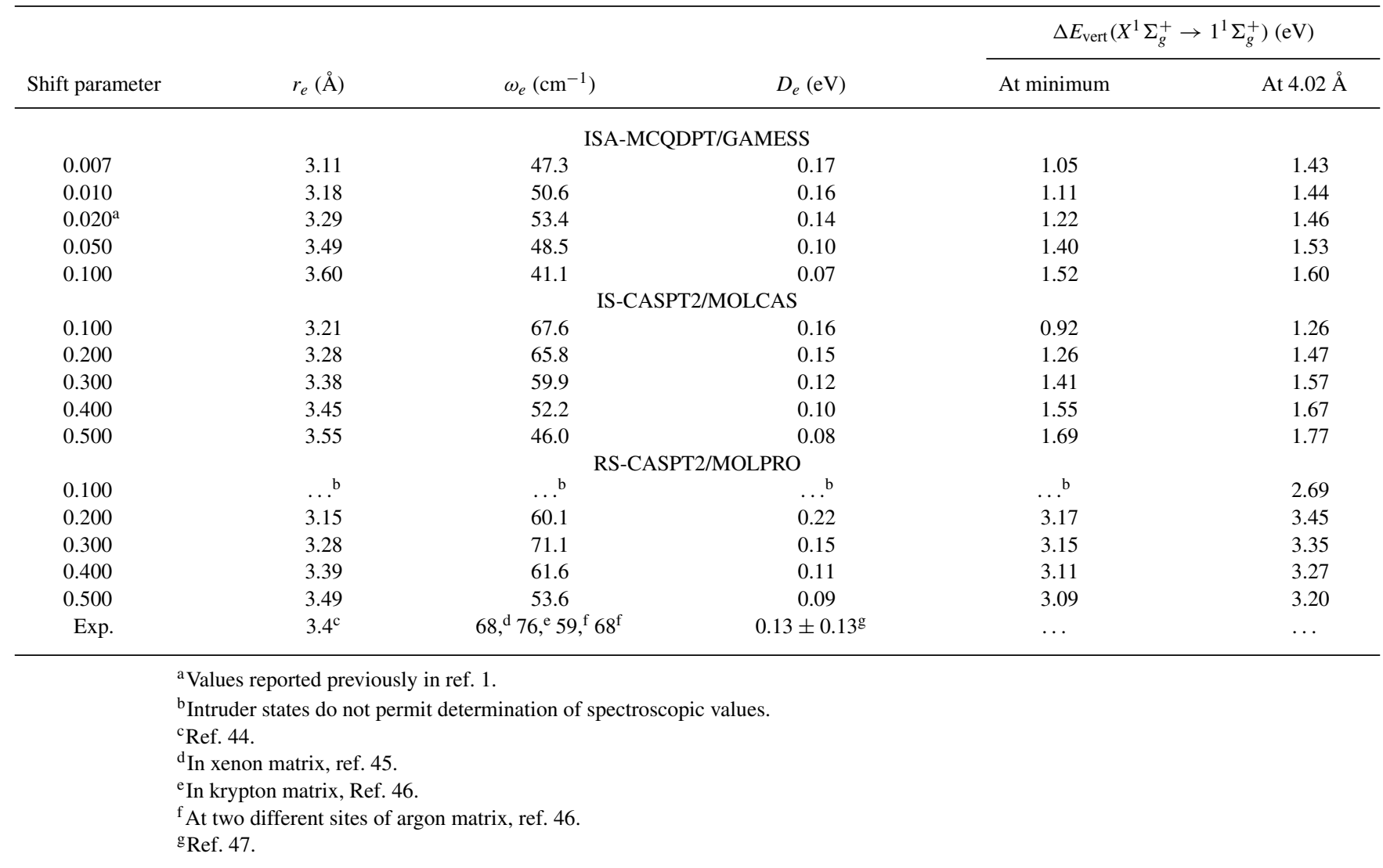

potential energy curves for the $\mathrm{Mn}_{2}$ ground state calculated using the resultant reduced atomic basis sets are shown in Figure 7 together with the original $(18 s 15 p 8 d 4 f 2 g) /[7 s 6 p 4 d 4 f 2 g]$ MCQDPT curve. It is clear from the curves presented in Figure 7 that the reduction of the atomic basis set is not a general remedy for the intruder state problem. The reduction of the one-particle basis allows for diminishing the number of intruder states, but at the same time leads to considerable reduction of the portion of dynamical correlation recovered by MCQDPT. At $4.0 \AA$, the reduced basis set calculations account only for the following fraction of the original MCQDPT correlation energy: $91.6 \%$ for the $(18 s 15 p 8 d 4 f) /[7 s 6 p 4 d 4 f]$ basis set, $54.7 \%$ for the $(18 s 15 p 8 d) /[7 s 6 p 4 d]$ basis set, and $53.3 \%$ for the $(18 s 13 p 8 d) /[7 s 4 p 4 d]$ basis set. For the smallest reduced basis, the MCQDPT curve is free from intruders around its minimum and therefore it is possible to determine the spectroscopic parameters for the $X^{1} \Sigma_{g}^{+}$state of $\mathrm{Mn}_{2}$. They are: $r_{e}=3.47 \AA, \omega_{e}=52 \mathrm{~cm}^{-1}$, and $D_{e}=0.07 \mathrm{eV}$. These values are comparable to those obtained previously $^{1}$ with ISA-MCQDPT $\left(r_{e}=3.29 \AA, \omega_{e}=54 \mathrm{~cm}^{-1}\right.$, and $\left.D_{e}=0.14 \mathrm{eV}\right)$ and from experiment ${ }^{44-46,48}\left(r_{e}=3.4 \AA, \omega_{e}=59\right.$, 68 , and $76 \mathrm{~cm}^{-1}$, and $\left.D_{e}=0.02-0.15 \mathrm{eV}\right)$.

\section{Modification of Orbital Canonicalization Scheme}

Two different orbital canonicalization schemes have been employed to test how the choice of the set of molecular orbitals influences the number of intruder states present in multistate MCQDPT calculations. The departure point for constructing these schemes is a set of state-averaged CASSCF orbitals. The canonical Fock matrix, given by

$$
F_{p q}=h_{p q}+\sum_{r s} D_{r s}\left[(p q \mid r s)-\frac{1}{2}(p r \mid q s)\right]
$$

where $D$ is a one-particle density matrix, is constructed and subsequently diagonalized in each of the three orbital subspaces (doubly-occupied, active, and virtual) yielding a set of canonical MOs and a set of orbital energies. The first canonicalization scheme uses the standard definition of the density matrix $D$ computed as an average over the three lowest CASSCF states with the $A_{g}$ symmetry. In the second canonicalization scheme $D$ is computed only from the ground state CASSCF wave function. The multistate MCQDPT potential energy curves obtained with the first canonicalization scheme are shown in the upper panel of Figure 4 and the PESs obtained with the second canonicalization scheme, in Figure 8 . Because both sets of curves are heavily deformed by numerous intruder states, the corresponding ISA-MCQDPT curves with the shift parameter of 0.02 are given for comparison. It is clear that the ground state PES obtained with the orbitals canonicalized only for the ground state (Scheme 2) is of better quality, because the 


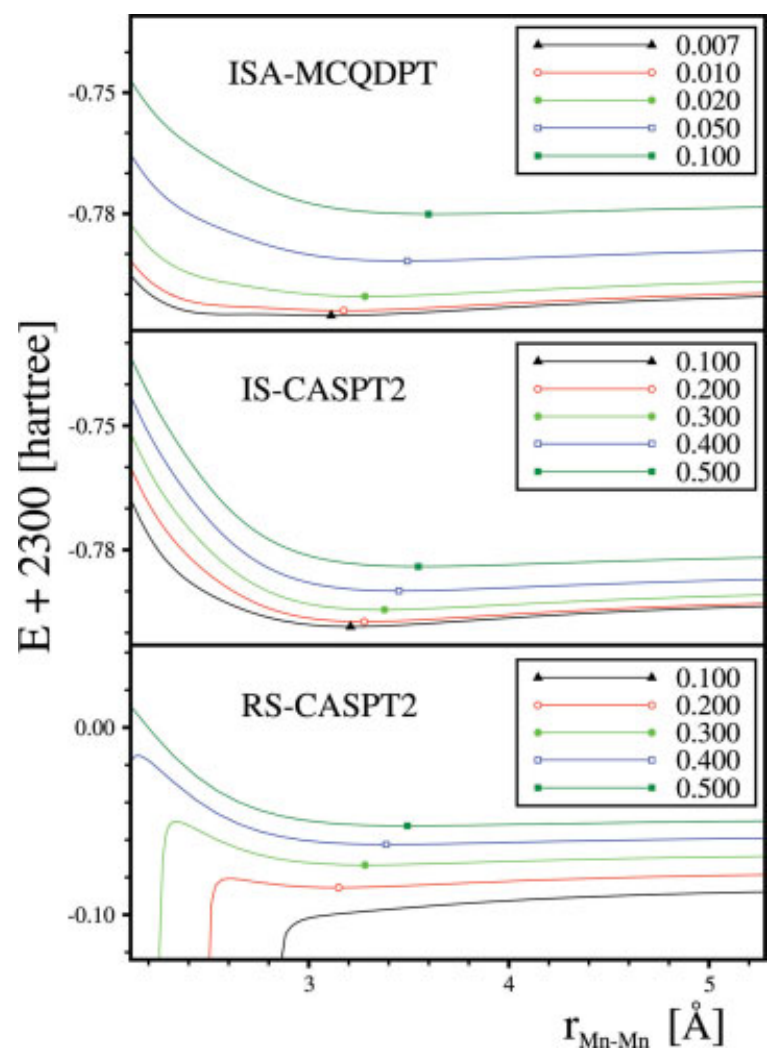

Figure 6. Potential energy curves for the $X^{1} \Sigma_{g}^{+}$state of $\mathrm{Mn}_{2}$ computed using various variants of MRPT with additional shift techniques. The presented curves correspond to different values of the shift parameter. Minimum of each curve is marked with a symbol. Note strong dependence of the position of the minima on the value of the shift parameter. [Color figure can be viewed in the online issue, which is available at www.interscience.wiley.com.] intruders are present only at short distances. This allows for finding spectroscopic parameters for the ground state PES computed with MCQDPT. They are: $r_{e}=3.31 \AA$, $\omega_{e}=56 \mathrm{~cm}^{-1}$, and $D_{e}=$ $0.13 \mathrm{eV}$.

\section{Modification of Effective MCQDPT Hamiltonian Dimension}

Previously reported MCQDPT calculations ${ }^{1}$ used the three lowest $A_{g}$ roots $-X^{1} \Sigma_{g}^{+}, 1^{1} \Sigma_{g}^{+}$, and a single component of $1^{1} \Delta_{g}$ - to define the effective MCQDPT Hamiltonian. Performing state-averaged CASSCF optimization of orbitals and averaging density matrices of three states in the canonicalization scheme may affect the number of intruder states present in the MCQDPT calculations. To test this dependence, we have performed two alternative sets of MCQDPT calculations: (a) with the lowest $A_{g}$ state $\left(X^{1} \Sigma_{g}^{+}\right)$and (b) with the two lowest $A_{g}$ states $\left(X^{1} \Sigma_{g}^{+}\right.$and $\left.1^{1} \Sigma_{g}^{+}\right)$. Figure 9 shows the potential energy curves obtained from these calculations. Modifying the dimension of the effective MCQDPT Hamiltonian could not prevent the zeroth-order spectrum quasidegeneracies completely, but the number of intruder states could be reduced, especially in case (a). Unfortunately, this MCQDPT curve displays another problem; the calculated PES is discontinuous at approximately $4.23 \AA$. The discontinuity is related to a switch of two orbitals between the active and inactive MO subspaces in the CASSCF calculations. No discontinuity is visible in the CASSCF curve, only accounting for dynamical correlation allows for noticing this deficiency. A more detailed analysis of this phenomenon is given in ref. 49. Additional discussion on the multistate MCQDPT calculations performed for the lowest ten $A_{g}$ states is given in Auxiliary Materials.

\section{Modification of Active Space}

Probably the most standard way of avoiding the intruder state problem in MRPT is an appropriate modification of the active space. Note

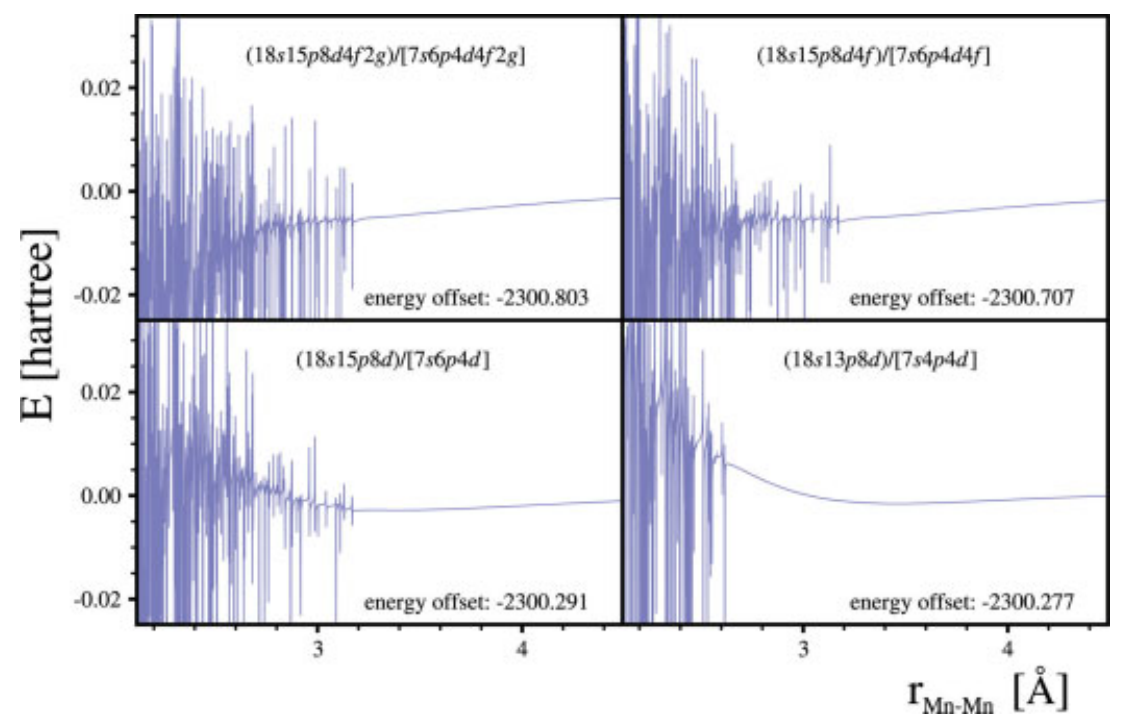

Figure 7. MCQDPT potential energy curves for the $X^{1} \Sigma_{g}^{+}$state of $\mathrm{Mn}_{2}$ computed with four 4 different basis sets. [Color figure can be viewed in the online issue, which is available at www.interscience.wiley.com.] 


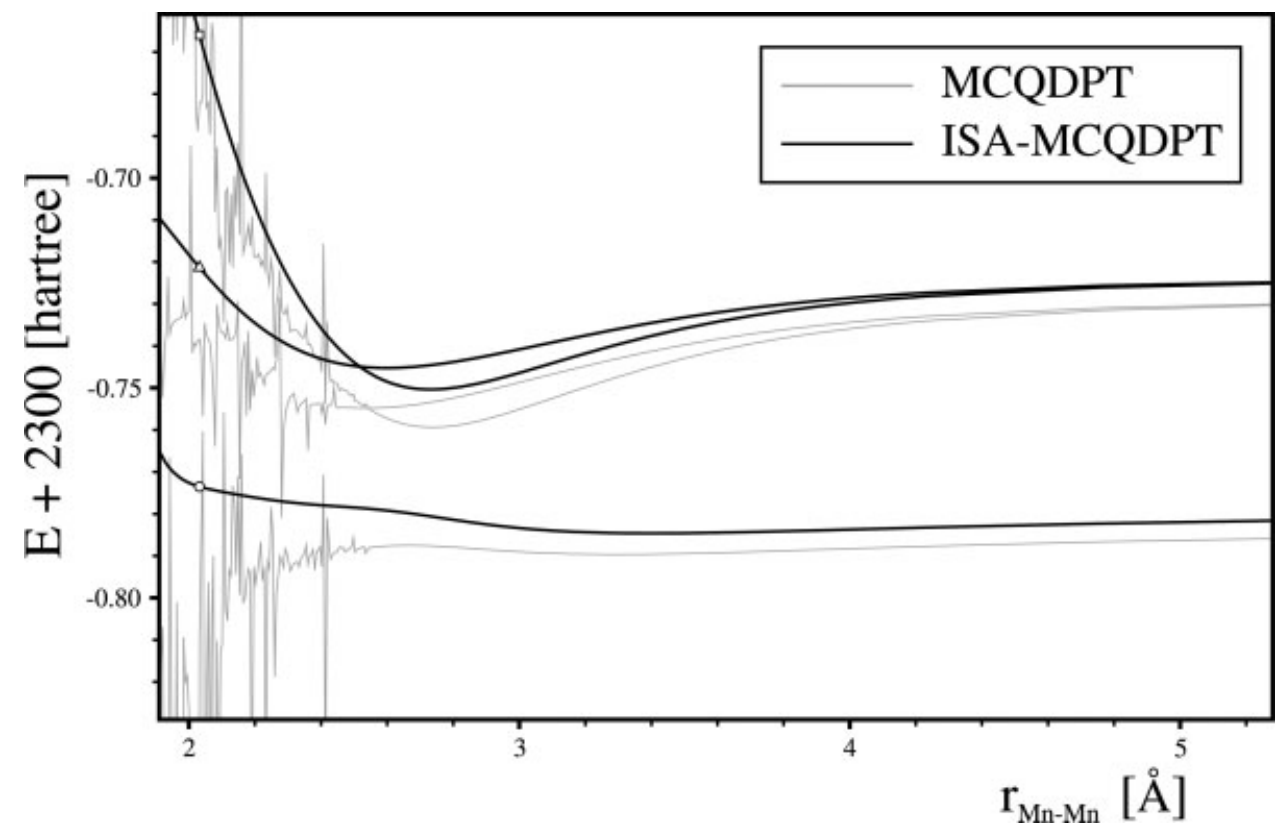

Figure 8. Comparison of the MCQDPT and ISA-MCQDPT potential energy curves for the $X^{1} \Sigma_{g}^{+}(\bigcirc)$, $1^{1} \Sigma_{g}^{+}(\square)$, and $1^{1} \Delta_{g}(\triangle)$ states of $\mathrm{Mn}_{2}$ computed with a set of orbitals canonicalized only for the ground state. Analogous curves computed with orbitals canonicalized over all studied states are shown in the upper panel of Figure 4. The value of the ISA shift parameter is 0.02 .

that in the studied case, including in the active space all low-lying virtual orbitals responsible for appearing of intruder states is infeasible since the dimension of the resulting CASSCF problem would be too large. Here, we test two alternative active spaces applicable to the multistate MRPT calculations for the ground state of manganese dimer. The first of the active spaces, denoted as $(12 \mathrm{o}, 12 \mathrm{e})$, is obtained by moving the $\sigma_{g}^{4 s}$ active orbital to the inactive subspace and by moving the $\sigma_{g}^{4 p}$ virtual orbital to the active subspace. The second active space, denoted as $(13 \mathrm{o}, 14 \mathrm{e})$, is obtained by moving the $\sigma_{g}^{4 p}$ virtual orbital to the active subspace. The MCQDPT potential energy curve for the first active space is calculated using two-state MCQDPT formalism $\left(X^{1} \Sigma_{g}^{+}\right.$and $\left.1^{1} \Sigma_{g}^{+}\right)$and for the second active space, using three-state MCQDPT formalism $\left(X^{1} \Sigma_{g}^{+}, 1^{1} \Sigma_{g}^{+}\right.$, and a single component of $1^{1} \Delta_{g}$ ). Both calculated curves, shown in Figure 10 , are affected by intruder states. The number of detected singularities is noticeably smaller than for the original $(12 \mathrm{o}, 14 \mathrm{e})$ active space, but on the other hand the singularities are present in a larger segment of the calculated PESs.

Several other choices of active space-including the $(12 \mathrm{o}, 12 \mathrm{e})$ and $(13 \mathrm{o}, 14 \mathrm{e})$ CAS studied here-are tested in ref. 49 in conjunction with the single-state MCQDPT formalism. None of the employed active spaces allows for avoiding the intruder state problem entirely, however, the observed intruders are present only at short interatomic distances. The computed curves are smooth around their minima allowing for determination of spectroscopic parameters for the ground state of $\mathrm{Mn}_{2}$. The equilibrium bond lengths are between 3.24 and $3.50 \AA$, the harmonic vibrational frequencies, between 44 and $72 \mathrm{~cm}^{-1}$, and the dissociation energies, between 0.05 and $0.09 \mathrm{eV}$. Because the coupling between the $X^{1} \Sigma_{g}^{+}$and $1^{1} \Sigma_{g}^{+}$states is relatively strong, it is possible that these values can be significantly altered in multistate MCQDPT calculations.

\section{Modification of the Zeroth-Order Hamiltonian}

Recently, a new definition of the zeroth-order Hamiltonian has been proposed for the CASPT2 method. ${ }^{50}$ In principle, it was introduced to diminish systematic errors observed for open shell systems, but it was also found to reduce significantly the intruder state problem. In the new zeroth-order Hamiltonian, a real shift-referred to as the IPEA shift-weighted by a function of the diagonal density matrix elements is used to correct the energies of active orbitals. The default value of the IPEA shift in the CASPT2/MOLCAS program is 0.25 hartree; this value was determined empirically for dissociation energies of 49 diatomic molecules. Applying the IPEA shift to the multistate CASPT2/MOLCAS calculations on $\mathrm{Mn}_{2}$ allows for significant improvement of the computed potential energy curves. Intruder states can be observed only at short distances. The ground state spectroscopic parameters determined from these calculations are: $r_{e}=4.23 \AA, \omega_{e}=29 \mathrm{~cm}^{-1}$, and $D_{e}=0.06 \mathrm{eV}$. Unfortunately, the computed spectroscopic parameters of $\mathrm{Mn}_{2}$ depend strongly on the value of the IPEA shift suggesting certain arbitrariness of the values presented earlier. Additional details of the IPEA-CASPT2 calculations are presented in Auxiliary Materials.

Summarizing the results obtained in this Section, we state that no standard technique of avoiding intruder states could be fully successfully employed to solve the intruder state problem in our multistate MRPT calculations on the ground state of $\mathrm{Mn}_{2}$. We have found that removing diffused atomic orbitals from the basis set, reducing the number of interacting states in the effective MCQDPT Hamiltonian, using the set of orbitals canonicalized only for the ground 


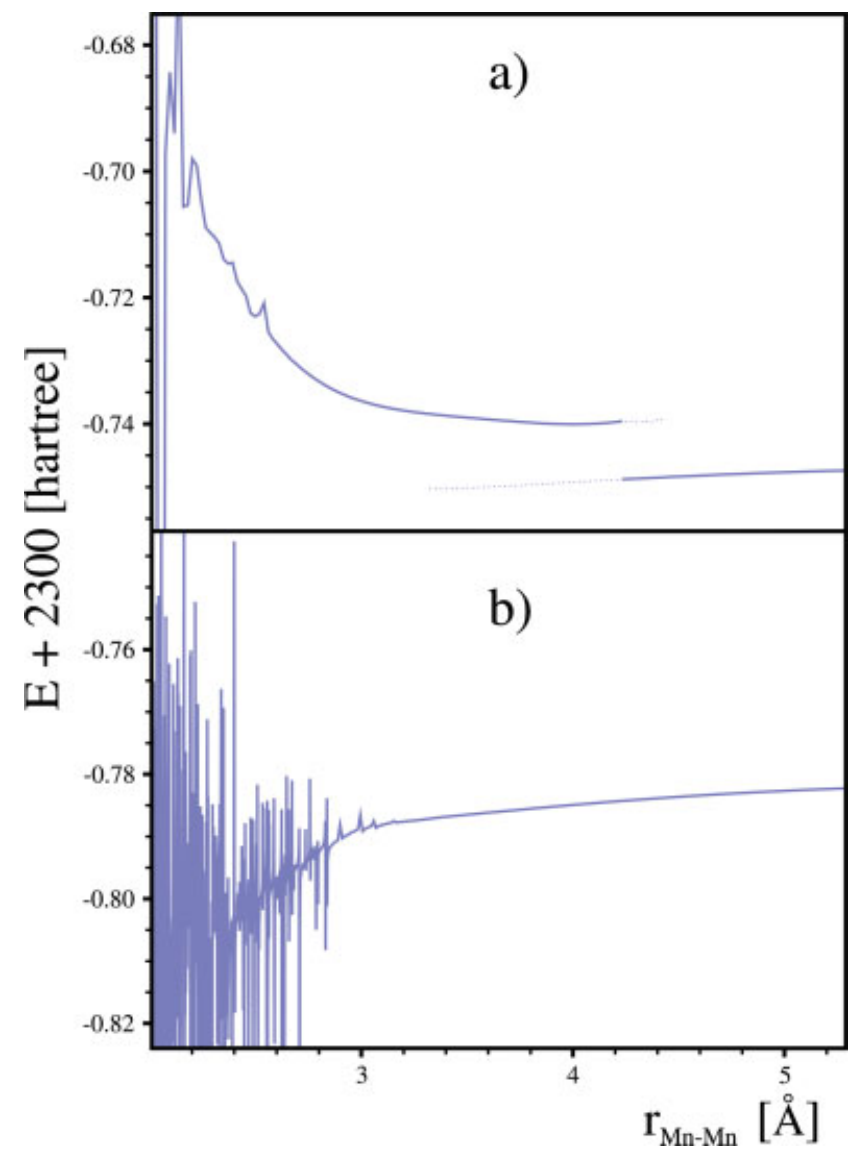

Figure 9. Potential energy curves for the $X^{1} \Sigma_{g}^{+}$state of $\mathrm{Mn}_{2}$ computed with (a) one-state and (b) two-state $(12 \mathrm{o}, 14 \mathrm{e})$ MCQDPT formalism. [Color figure can be viewed in the online issue, which is available at www.interscience.wiley.com.]

state, or including low-lying virtual orbitals in the active space helps to reduce the extent of the intruder state problem, however, none of these techniques is able to remove it entirely. Probably, the most promising approach is to use the IPEA shift in the definition of the modified zeroth-order Hamiltonian. Strong dependence of the computed spectroscopic parameters on the value of the IPEA shift suggests that this issue requires further clarification.

\section{Conclusion}

A detailed, spatially-resolved analysis of the previously reported ${ }^{1}$ multistate MCQDPT potential energy curve for the $X^{1} \Sigma_{g}^{+}$state of manganese dimer is presented. We show that the computed PES suffers from numerous (more than 5000!) discontinuities, which originate from quasidegeneracies in the zeroth-order Hamiltonian spectrum. The one- and two-electron excited intermediate states that are responsible for appearance of these intruder states are identified and analyzed. These states belong to two classes of excitations: single and double excitations from active orbitals to external orbitals. Additional calculations performed with other versions of multistate MRPT (fully- and partially-contracted CASPT2) show that the reported serious intruder state problem is not only specific to the MCQDPT method, but also it is rather a common problem of MSMRPTs. Using standard shift techniques in the multistate MRPT formalism allows for removing all intruders, but unfortunately, it also introduces a large arbitrariness in the calculated results. The shape of the potential energy curve and the values of the spectroscopic parameters of the $X^{1} \Sigma_{g}^{+}$state of $\mathrm{Mn}_{2}$ are found to depend strongly on the value of shift parameters. These findings rise serious controversies regarding validity of employing shift techniques for solving the intruder state problem in MRPT. A set of various alternative methods of removing intruder states is tested: removing diffused atomic orbitals from the basis set, reducing the number of interacting states in the effective MCQDPT Hamiltonian, using the set of orbitals canonicalized only for the ground state, including low-lying virtual orbitals in the active space, and modifying the zeroth-order Hamiltonian. None of these tested alternative approaches is able to completely eliminate the intruder state problem, but some of these approaches could reduce its severity considerably. Probably the most promising approach is using the zeroth-order Hamiltonian modified with the IPEA shift.

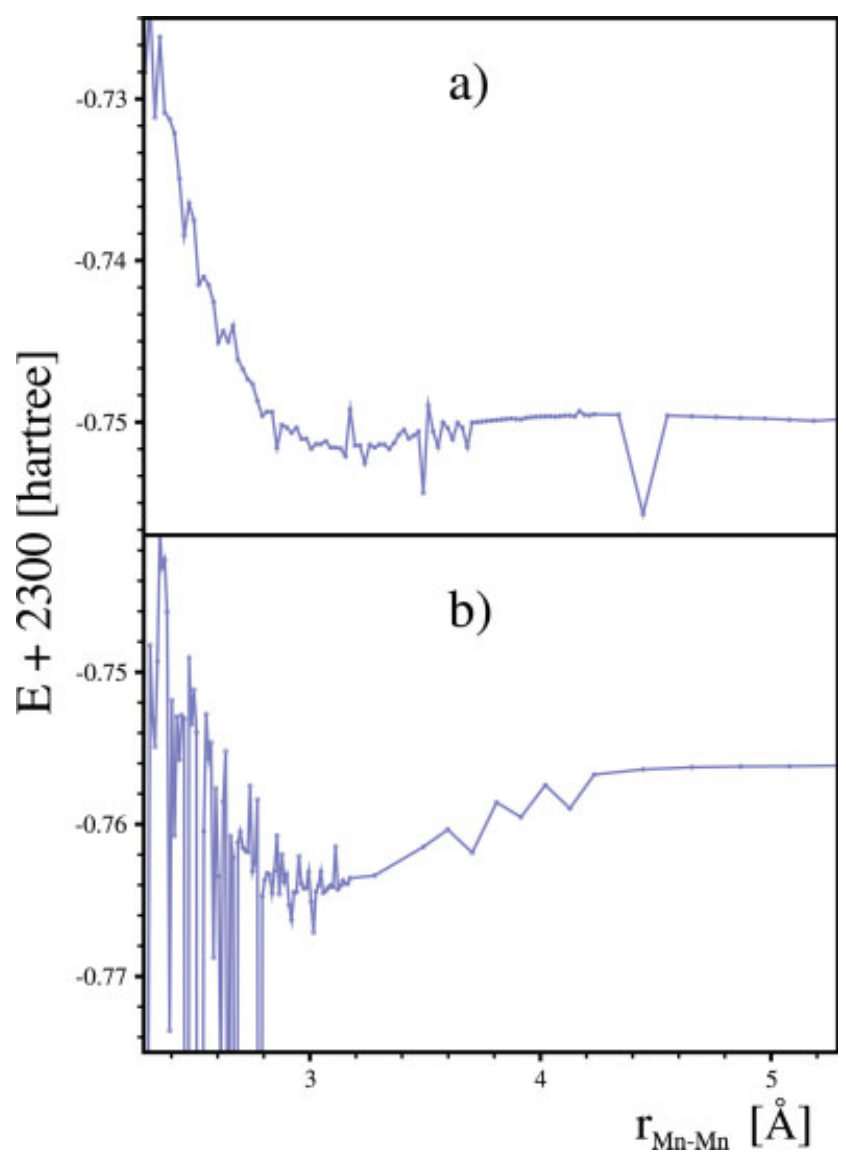

Figure 10. Potential energy curves for the $X^{1} \Sigma_{g}^{+}$state of $\mathrm{Mn}_{2}$ computed with (a) two-state $(120,12 \mathrm{e}) \mathrm{MCQDPT}$ and with (b) three-state $(13 \mathrm{o}, 14 \mathrm{e}) \mathrm{MCQDPT}$. [Color figure can be viewed in the online issue, which is available at www.interscience.wiley.com.] 
We believe that the lowest three $A_{g}$ states of manganese dimer constitute a perfect benchmark case for studying the behavior of MRPT in extreme situations. The problems detected and communicated in this article can provide an invaluable benchmark framework for development of reliable and computationally stable MS-MRPT. We also hope that the discovered strong dependence of the computed potential energy curves and the spectroscopic parameters on the value of the shift parameter will issue a warning for all users of MRPT that use the shift techniques in a completely automated fashion to solve the intruder state problem. ${ }^{25-39}$ We advocate here using two different shift parameters and comparing the resulting data. If the results are similar, it is safe to assume that the employed shift technique does not have large influence on the calculated results.

\section{References}

1. Yamamoto, S.; Tatewaki, H.; Moriyama, H.; Nakano, H. J Chem Phys 2006, 124, 124302.

2. Wang, B.; Chen, Z. Chem Phys Lett 2004, 387, 395.

3. Wang, B.; Chen, Z. J Chem Phys 2005, 123, 134306.

4. Bauschlicher, C. W. Jr. Chem Phys Lett 1989, 156, 95.

5. Nesbet, R. K. Phys Rev A 1964, 135, 460.

6. Harris, J.; Jones, R. O. J Chem Phys 1979, 70, 830.

7. Pederson, M. R.; Reuse, F.; Khanna, S. N. Phys Rev B 1998, 58, 5632.

8. Desmarais, N.; Reuse, F. A.; Khanna, S. N. J Chem Phys 2000, 112, 5576.

9. Yanagisawa, S.; Tsuneda, T.; Hirao, K. J Chem Phys 2000, 112, 545.

10. Barden, C. J.; Rienstra-Kiracofe, J. C.; Schaefer, H. F. III, J Chem Phys 2000, 113, 690 .

11. Bobadova-Parvanova, P.; Jackson, K. A.; Srinivas, S.; Horoi, M. J Chem Phys 2005, 122, 014310.

12. Valiev, M.; Bylaska, E. J.; Weare, J. H. J Chem Phys 2003, 119, 5955.

13. Gutsev, G.; Bauschlicher, C. J Phys Chem A 2003, 107, 4755.

14. Nayak, S. K.; Jena, P. Chem Phys Lett 1998, 289, 473.

15. Fujima, N.; Yamaguchi, T. J Phys Soc Jpn 1995, 64, 1251.

16. Yamanaka, S.; Ukai, T.; Nakata, K.; Takeda, R.; Shoji, M.; Kawakami, T.; Takada, T.; Yamaguchi, K. Int J Quantum Chem 2007, 107, 3178.

17. Yamaguchi, K.; Tsunekawa, T.; Toyoda, Y.; Fueno, T. Chem Phys Lett 1988, 143, 371 .

18. Witek, H. A.; Choe, Y.-K.; Finley, J. P.; Hirao, K. J Comput Chem 2002, 23, 957.

19. Forsberg, N. Malmqvist, P. Å. Chem Phys Lett 1997, 274, 196.

20. Khait, Y. G.; Song, J.; Hoffmann, M. R. J Chem Phys 2002, 117, 4133.

21. Roos, B. O.; Andersson, K. Chem Phys Lett 1995, 245, 215.

22. Schmidt, M. W.; Baldridge, K. K.; Boatz, J. A.; Elbert, S. T.; Gordon, M. S.; Jensen, J. H.; Koseki, S.; Matsunaga, N.; Nguyen, K. A.; Su, S.; et al. J Comput Chem 1993, 14, 1347.

23. Werner, H.-J.; Knowles, P. J.; Lindh, R.; Manby, F. R.; Schütz, M.; Celani, P.; Korona, T.; Rauhut, G.; Amos, R. D.; Bernhardsson, A.;
Berning, A.; Cooper, D. L.; Deegan, M. J. O.; Dobbyn, A. J.; Eckert, F.; Hampel, C.; Hetzer, G.; Lloyd, A. W.; McNicholas, S. J.; Meyer, W.; Mura, M. E.; Nicklass, A.; Palmieri, P.; Pitzer, R.; Schumann, U.; Stoll, H.; Stone, A. J.; Tarroni, R.; Thorsteinsson, T. Molpro, version 2006.1, A Package of Ab initio Programs, 2006. http://www.molpro.net.

24. Karlstrom, G.; Lindh, R.; Malmqvist, P. Å.; Roos, B. O.; Ryde, U.; Veryazov, V.; Widmark, P.-O.; Cossi, M.; Schimmelpfennig, B.; Neogrady, P.; Seijo L. Comput Mater Sci 2003, 28, 222.

25. Fedorov, D. G.; Nakajima, T.; Hirao, K. J Chem Phys 2003, 118, 4970.

26. Iuchi, S.; Morita, A.; Kato, S. J Chem Phys 2004, 121, 8446.

27. Patchkovskii, S. Phys Chem Chem Phys 2006, 8, 926.

28. Sliznev, V. V.; Vogt, N.; Vogt, J. J Mol Struct 2006, 780-781, 247.

29. Gopakumar, G.; Lievens, P.; Nguyen, M. T. J Chem Phys 2006, 124, 214312.

30. Doyle, R. J.; Campo, R. D.; Taylor, P. R.; Mackenzie, S. R. J Chem Phys 2004, 121, 835 .

31. Pierloot, K.; Vancoillie, S. J Chem Phys 2006, 125, 124303.

32. Oliver, T. A. A.; Taylor, P. R.; Doyle, R. J.; Mackenzie, S. R. J Chem Phys 2007, 127, 024301.

33. Olaso-González, G.; Roca-Sanjuán, D.; Serrano-Andrés, L.; Merchán, M. J Chem Phys 2006, 125, 231102.

34. Zou, W.; Liu, W. J Chem Phys 2006, 124, 154312.

35. Chen, H.; Li, S. J Chem Phys 2006, 124, 154315.

36. Pierloot, K.; van Besien, E. J Chem Phys 2005, 123, 204309.

37. de Graaf, C.; Hozoi, L.; Broer, R. J Chem Phys 2004, $120,961$.

38. Pierloot, K.; Delabie, A.; Groothaert, M. H.; Schoonheydt, R. A. Phys Chem Chem Phys 2001, 3, 2174.

39. Rubio, M.; Roos, B. O.; Serrano-Andrés, L.; Merchán, M. J Chem Phys 1999, 110, 7202.

40. Choe, Y.-K.; Witek, H. A.; Finley, J. P.; Hirao, K. J Chem Phys 2001, $114,3913$.

41. Nakano, H. J Chem Phys 1993, 99, 7983.

42. Andersson, K.; Malmqvist, P. Å.; Roos, B. O.; Sadlej, A. J.; Wolinski, K. J Phys Chem 1990, 94, 5483.

43. Andersson, K.; Malmqvist, P. Å.; Roos, B. O. J Chem Phys 1992, 96, 1218.

44. Baumann, C. A.; Van Zee, R. J.; Bhat, S. V.; Weltner, W. Jr. J Chem Phys 1983, 78, 190.

45. Bier, K. D.; Haslett, T. L.; Kirkwood, A. D.; Moskovits, M. Chem Phys Lett 1988, 89, 6.

46. Kirkwood, A. D.; Bier, K. D.; Thompson, J. K.; Haslett, T. L.; Huber, A. S.; Moskovits, M. J Phys Chem 1991, 95, 2644.

47. Kant, A.; Lin, S.; Strauss, B. J Chem Phys 1968, 49, 1983.

48. Haslett, T. L.; Moskovits, M.; Weitzman, A. J Mol Spectrosc 1989, 135, 259.

49. Camacho, C.; Yamamoto, S.; Witek, H. A. Phys Chem Chem Phys (in press), DOI:10.1039/B805125A.

50. Ghigo, G.; Roos, B. O.; Malmqvist, P. Å. Chem Phys Lett 2004, 396, 142 . 